\title{
Correction to: Proper affine actions: a sufficient criterion
}

\author{
Ilia Smilga ${ }^{1}$
}

Published online: 17 November 2021

(c) Springer-Verlag GmbH Germany, part of Springer Nature 2021

\section{Correction to: Mathematische Annalen https://doi.org/10.1007/s00208-020-02100-7}

In the orginal article, the "only if" part of Proposition 6.15 is obviously false. In fact, the condition of $Y$ being "compatible with $X_{0}$ " as defined here is stronger than the condition of both $g$ and $g^{-1}$ being simultaneously contracting.

To correct this mistake, it suffices to replace Definition 6.12.(iii) by the following one:

Definition 6.12 (Affine version of regularity). We say that a vector $Y \in \mathfrak{a}$ is:

[...]

(iii) compatible with $X_{0}$ if

$$
\forall \lambda^{<} \in \Omega_{X_{0}}^{<}, \quad \forall \lambda^{=} \in \Omega_{X_{0}}^{=}, \quad \forall \lambda^{>} \in \Omega_{X_{0}}^{>}, \quad \lambda^{<}(Y)<\lambda^{=}(Y)<\lambda^{>}(Y) .
$$

Proposition 6.15 then effectively becomes a tautology. On the other hand, the first sentence of Remark 6.14 only applies to the stronger (wrong) notion of compatibility with $X_{0}$; with the correct definition, it should just be discarded. All of the other results of the paper remain true, with the correct definition in mind.

Let us also draw the reader's attention to the set $\mathfrak{a}_{\rho, X_{0}}^{\prime}$ of vectors compatible with $X_{0}$, whose definition changes accordingly. The wording of its definition (Definition 6.20) does not need to be changed, as it directly relies on Definition 6.12.

This mistake also indirectly affects my subsequent paper [1], which uses the set $\mathfrak{a}_{\rho, X_{0}}^{\prime}$, for whose definition it explicitly refers to Definition 6.20. The correct definition to be used is of course the one given in the present corrigendum. Having this in mind, no other modifications to the text of [1] are then needed.

The original article can be found online at https://doi.org/10.1007/s00208-020-02100-7.

$凶 \quad$ Ilia Smilga

ilia.smilga@normalesup.org

1 Institut des Hautes Études Scientifiques et CNRS, 35 route de Chartres, 91440 , Bures-sur-Yvette, France 


\section{References}

1. Smilga, I.: Construction of Milnorian representations. Geom. Dedicata 206, 55-73 (2020)

Publisher's Note Springer Nature remains neutral with regard to jurisdictional claims in published maps and institutional affiliations. 